\title{
The Indeterminate Present and the Open Future
}

\author{
Cristian Mariani - Giuliano Torrengo
}

Penultimate version.

Final version published for Synthese: https://doi.org/10.1007/s11229-020-02963-y

\begin{abstract}
Explanations of the genuine openness of the future often appeal to objective indeterminacy. According to the received view, such indeterminacy is indeterminacy of certain future-tensed state of affairs that presently obtain. We shall call this view the weak indeterminate present, to distinguish it from the view we will defend in this paper, which we dub the strong indeterminate present. According to our view, unsettledness of the future is grounded on the present indeterminacy of some present-tensed state of affairs. In order for an indeterminate present-tensed state of affairs to explain the unsettledness of a future-tensed state of affairs, there has to be a connection between the two. We argue that this connection can only be provided if we look at the internal structure of the relevant state of affairs. Finally, we will suggest that the best background theory to explain the connection are the so-called spontaneous collapse models of quantum mechanics.
\end{abstract}

Keywords Metaphysical Indeterminacy · Open Future · Quantum Mechanics · Spontaneous Collapse Models

Cristian Mariani acknowledges the generous support of the French National Research Agency, under the program Investissements d'avenir (ANR-15-IDEX-02). Giuliano Torrengo acknowledges the generous support of the Spanish Ministry of Science and Innovation (PID2019-108762GB-I00).

Cristian Mariani

Institut Néel (CNRS) \& Chair of Excellence in Philosophy of Quantum Physics, Université Grenoble Alpes

E-mail: cristian.mariani@neel.cnrs.fr

Giuliano Torrengo

State University of Milan \& Autonomous University of Barcelona

E-mail: giuliano.torrengo@uab.cat 


\section{Introduction}

The paper is structured as follows. In section 2 and 3, we analyse the received view on the relationship between openness of the future and indeterminacy in reality, along with the notion of weak indeterminate present (WIP) on which it is based. Section 4 gives the general outline of a theory of openness of the future based on the notion of strong indeterminate present (SIP). In section 5 , we focus on those features of the spontaneous collapse models of quantum mechanics that makes it the best candidate for a background theory to explain the openness of the future in terms of SIP, while in 6 we outline such connection in details. Section 7 concludes.

\section{Openness and Indeterminacy}

Among our ordinary intuitions about time, the idea of an asymmetry between past and future is perhaps the most pervasive, and yet a very mysterious one. We think of the past as fixed, no longer available to modification, and of the future as open, not yet settled. This pre-theoretical notion of openness is supported or suggested by a large number of phenomena. Firstly, our sense of agency, the feeling that we can contribute to make things happen in the future, as opposed to our sense of impotence towards the past, suggests it. Secondly, memory and expectation are two intentional states with different normative profiles. Memory is closely linked to its past target, and memory reports allows only for factive readings: in order for me to remember something the content of my memory has to represent correctly what happened in the past. ${ }^{1}$ Contrariwise, expectation does not require a determinate connection with the future, and expectation claims allows for a non-factive reading: in order for me to expect that $p$ will be the case, I do not need to correctly represent the future. Finally, although philosophers have tried to explain the temporal asymmetry between past and future in terms of the asymmetry between cause and effect (e.g., [43], it is quite natural to think of the difference between the cause and the effect as grounded in their difference in temporal location: effects are less determinate because they are not yet there, when the cause is (see [39]).

We all seem to agree that the notion of openness is somehow related to these phenomena. Disagreement comes when we try to provide a theoretical explanation of the notion. For instance, one could think that openness can be explained ontologically by certain theories of time. According to presentism (e.g., [27]) and to the growing block view (e.g., [17]), the future simply is not there, and that is the reason why it is open. Or we can think that openness is explained by there being a multiplicity of options and no fact of the matter at present with respect to which will be the actual one (e.g., [6]). Other views seek an explanation of openness in the metaphysics of passage. If there is genuine

\footnotetext{
1 Notice that the fact that memory has such a normative aspect is compatible with the fact that psychologists are usually interested in the cognitive mechanisms underlying memory regardless of their truthfulness. See [7].
} 
becoming, then we can ground the asymmetry between fixity of the past and openness of the future on the directionality of flow (e.g., [14]).

Finally, certain views try to look at the laws of physics for an answer. If laws of physics are fundamentally indeterministic, then perhaps openness is just a result of the fact that nature does not determine or fix all the future facts ([29], p. 38). This approach in particular has been widely discussed in the philosophy of physics literature, especially about quantum mechanics. Many physicists and philosophers in the past decades have argued, in one way or the other, that quantum mechanics supports the open future intuition, since its laws (at least on some interpretation) are fundamentally stochastic. However, as argued by many scholars the above line of reasoning is not as straightforward as it might seem, at least for two reasons. First, as it has been argued (see [35]), indeterministic laws would not just make the future open, but would make the past open as well, thus in fact leading to a clash with the asymmetry intuition. ${ }^{2}$ Second, and perhaps more importantly, there is the issue nicely summarized by Pooley $^{3}$ in the passage below:

Suppose that the laws of nature are indeterministic in the sense that specification of the world's history up to a certain time, together with those laws, does not fix all future facts. To say that the future is open might only be to say that the future is not nomologically determined in this sense. But that the past and present, together with the laws, do not fix all future facts does not entail that there are no such facts. In tenseless terms, there can be a unique actual continuation of the world to the future of some time $t$, but this continuation need not be the only one compatible with the actual laws and the way the world is up to and including $t .([42]$, p. 322)

The mere fact that indeterministic laws of nature - together with all the facts about what is present and what is past - do not determine a unique future, does not mean that there is no such future. Whether or not the laws entail a unique future, from a metaphysical perspective there still remains the problem of the different ontological status of the future with respect to the present and the past.

In order to assess whether QM supports the open future thesis, perhaps a better starting point would be to find what could be a minimal theoretical consensus on the notion of openness, one that is shared by everybody who participates in this debate. If the future is open, and the universe shows some qualitative diversity through time, contingent matters about the future are unsettled. Traditionally, this idea is captured by appealing to the notion of

\footnotetext{
2 This may not be a fatal strike to the proposal. After all, one may follow [33] and argue that if our best account of the openness of the future comes with a confutation of the asymmetry, so much the worst for the latter. In what follows we will put on a side this option, since our focus is over a view of openness that aims to preserve the asymmetry intuition.

3 See also [47] and [11] for similar conclusions, though with a focus on the the so-called thin red line view.
} 
future contingents, that is future-tensed claims that are neither necessarily true (as "tomorrow either it will rain or it will not" is) nor necessarily false (as "tomorrow both it will rain and it will not" is). A mark or symptom of the openness of the future is thus that future contingents lack a determinate truth value. ${ }^{4}$ We take such a mark to be a crucial desideratum, that is, any theory of openness should abide by the adequacy condition $\mathrm{AC}$ below.

(AC) Assuming that there is some qualitative variation across time, future contingents are unsettled with respect to their determinate truth value

In other words, we could assume that in order for a theory of the openness of the future to be adequate, it has to entail that the truth or falsity of propositions $^{5}$ like $<$ There will be a protest in front of Wall Street tomorrow $>$ is not at present settled. But what do we mean by unsettledness here, and what is precisely the link between unsettledness of future contingents and openness of the future? This question is particularly pressing since, after all, one could have a merely epistemic take on unsettledness, and claim that the only reason why future contingents are unsettled is because we just do not know yet whether a certain proposition is true or false. However, if our aim is to characterize the idea of the openness of the future as something not entirely due to our ignorance, clearly an epistemic take on the notion of unsettledness is uninteresting. If the future is open in a more robust and genuine sense, the unsettledness of future contingents have to be interpreted metaphysically, as an objective feature of the external world. That is to say, the world itself has to be unsettled with respect to the truth of future-tensed claims. What does an explanation of the temporal asymmetry in terms of a theory of metaphysical unsettledness of future contingents look like? And more generally, what sense, if any, can we make of the notion of metaphysical unsettledness?

The best way to think of unsettledness in a metaphysical way is by referring to the notion of indeterminacy, which appears to be more well regimented. If we grant this, ${ }^{6}$ then the openness of the future can be understood as a form

\footnotetext{
4 Notice that failure of having assigned at a time a determinate truth value does not necessarily entail failure of bivalence. See, e.g.: [37], [26], [3]. For instance, views that encompasses a thin red line (cf. [40]) maintain bivalence within a branching framework. In the rest of the paper, for simplicity's sake, we will talk about unsettledness as if it implies failure of bivalence.

5 A claim is the utterance of a sentence in a specific context, and claims usually express propositions. Propositions aim at representing state of affairs, which are what make them true, when they are true. We use the notation " $<\ldots>$ " for propositions, and the notation "[...]" for states of affairs. We take the notion of being tensed to be applied to sentences, but also, derivatively, to the claims made by using sentences, to the propositions expressed, and to the state of affairs they aim at representing.

6 Notice that, if you are already committed to accept metaphysical unsettledness, then accepting metaphysical indeterminacy does no harm. The use of the notion of metaphysical indeterminacy just is the best way to spell out a prior commitment towards taking unsettledness in a robust way. Barnes \& Cameron ([3], p. 304) admit that the acceptance of metaphysical indeterminacy in order to defend the open future might be taken as a cost. However, they correctly rejoin that their proposal "is concerned with someone who has already accepted the open future thesis. She is therefore already committed to making sense
} 
of objective indeterminacy. More precisely, a natural idea is to think that a certain kind of objective indeterminacy of the future explains why future contingents are now unsettled. Thus, the order of explanation goes from an objectively indeterminate future to the unsettledness of future-tensed claim when evaluated at an objectively determinate present. Future contingents are "oriented" toward the future (their representational aims lie in the future), but are evaluated as unsettled in the present. What is unsettled is in the present (the truth value of future contingents, or the result of their evaluation), what is indeterminate is in the future (its openness), and future indeterminacy explains present unsettledness.

Notice, though, that indeterminacy of the future cannot mean that when the future arrives we will find that it is indeterminate. The indeterminacy of the future is resolved when the future becomes present. So, it seems prima facie contradictory to say that there is indeterminacy in the future, since the future is not indeterminate when it becomes present. Rather, the indeterminacy is in the future only until it does not arrive - it is from the point of view of the present, so to say, that the future lacks determination. Per se, the future is as determinate as the present.

To sum up. The unsettledness of future contingents is the mark of the openness of the future. Such a mark is explained by the indeterminacy of the future, which is however indeterminate only as of the present. The question before us is then the following: if the future is indeterminate only as of the present (because when the future arrives, it will not be any longer indeterminate), does the openness of the future require also some form of indeterminacy in the present?

In what follows we will argue that according to what we call the received view, the present is indeterminate only in a weak sense, and we will thus dub this view the Weak Indeterminate Present (WIP). WIP is a weak form of indeterminacy of the present because no present-tensed states of affairs are indeterminate on this view, nor it is indeterminate which present-tensed states of affairs presently obtain. According to WIP, the openness of the future requires only a weak form of indeterminacy in the present, one concerning only which future-tensed states of affairs presently obtain. In the following sections, we will put this view in contrast with the view we call Strong Indeterminate Present (SIP), according to which the future is open because there is indeterminacy in certain present-tensed state of affairs. The connection between the indeterminate states of affairs in the present, and the indeterminacy of the future is given by the structural relations between the states of affairs. Roughly, the present-tensed indeterminate states of affairs are such that they can be "resolved" in more than one way in the future.

To give an example, we could think that the indeterminacy of $<$ There will be a protest in front of Wall Street tomorrow $>$ is grounded on the indeterminacy of some relevant present-tensed states of affairs that obtain at present,

of robust, non-representational indeterminacy (indeterminacy in how things are, rather than how they are described). In her case, she is committed to worldly indeterminacy concerning the future." 
such as the uncertainty among the members of the Congress relative to the upcoming vote, and the swaying feelings of the protesters. The reason why there is such a connection is that protests in front of Wall Street usually require a certain kind of motivation and attitudes, and how the present indeterminacy in the state of mind of the relevant persons will be resolved determines whether the protest takes place or not.

In the next section, we will provide an analysis of WIP, before moving to SIP in the rest of the paper.

\section{The Locus of Indeterminacy in the Received View}

A future contingent is a proposition expressed by a claim about some future contingent matter. If now, in 2020, you utter the future-tensed sentence "There will be a space battle around Terok Nor in four hundred years," you express a future contingent. In the standard analysis, the proposition expressed by your utterance is composed by a kernel present-tense proposition $<$ There is a space battle around Terok Nor $>$, the future sentential operator It will be the case that, and the metric determination (attached to the sentential operator) in four hundred years. In other words, by claiming that there will be a space battle around Terok Nor in four hundred years, I express the following proposition.

Space-BATtle < It will be the case (in four hundred years) that there is a space battle around Terok Nor $>$

Space-BAttle is clearly, in some sense, unsettled. For one thing, it is epistemically unsettled: we do not know yet if a battle around Terok Nor will take place in four hundred years or not. Besides, nobody would think that if SpACE-BATTLE is unsettled, then when four hundred years elapse, it is indeterminate whether a space battle is going on or not around Terok Nor. As already stressed, the future is not unsettled in this sense. Indeterminacy about the future is not indeterminacy in the future, for the indeterminacy will be resolved when the future arrives. Rather, everybody agrees that, if SPACEBATTLE is unsettled, then it is now indeterminate what will be the case with respect to space battles around Terok Nor in four hundred years. Openness of the future is indeterminacy of the future as of the present: the future is indeterminate as of now, not as of then.

Therefore, openness of the future requires some form of indeterminacy in the present. However, in the received view, such indeterminacy does not require the present to be constituted by any indeterminate state of affairs. We can introduce the notion of constitution as explained below.

Constitution A state of affairs partially constitutes a time $t$ if and only if it obtains at $t$ and it is exclusively about what is going on at $t$

The notion of "being about" is notoriously a vague one, but for our purposes suffice it to say that no future-tensed state of affairs that obtain at the present 
time is about the present time; they are all about what will be the case at future times. Thus, no future-tensed state of affairs can constitute the present. ${ }^{7}$

Since according to the received view the present is not constituted by indeterminate state of affairs, the openness of the future does not require indeterminacy in presently obtaining present-tensed state of affairs. Rather, the received view is that there is in the present indeterminacy with respect to future-tensed states of affairs. More precisely, it is at present indeterminate which of two incompatible states of affairs about a contingent course of event will take place. For instance, let us assume that time $t^{\prime}$ comes four hundred years later than time $t$. At $t$, it is indeterminate whether the state of affairs that [It will be the case in four hundred years that there is a space battle around Terok Nor] or the state of affairs that [It will be the case in four hundred years that there is no space battle around Terok Nor] obtains. The indeterminacy, at $t$, as to which state of affairs will obtain, explains why SPACE-BATTLE is in turn unsettled. ${ }^{8}$

Now, assuming that time $t$ is present, we can ask whether the indeterminacy as to which of the two state of affairs obtain makes the present indeterminate in some robust sense? There are good reasons to answer negatively. In order for the present to be indeterminate in some robust sense, it has to be constituted by indeterminate state of affairs, namely certain present-tensed state of affairs have to be indeterminate, or there has to be indeterminacy with respect to which present-tensed state of affairs obtain. Future-tensed state of affairs "point beyond themselves", as Sider (cf. [44]) puts it. Whether they obtain or not in the present depends on what will be the case later on, and not-not entirely at least - on what is going on in the present. Thus, the indeterminacy concerning which future-tensed state of affairs obtain at $t$ in the case of the space battle is not due to indeterminacy in $t$. This suggests that according to the received view the locus of indeterminacy should be in the future of $t$. Yet, the future is indeterminate only insofar as it is still future: it is at present indeterminate what will be the case.

7 The notion of constitution that we use is not innocent and could meet some resistance. Someone believing in irreducibly future-tensed properties may want to have the present constituted by future-tensed state of affairs or facts too. In the literature, Lucretian presentism (cf. [8]) is probably the position less sympathetic to our characterisation of constitution. Roughly, according to the Lucretian, present truths about the past are made truth by presently obtaining states of affairs involving the world and past-tensed properties. For instance, that Cesar crossed the Rubicon is made true by the presently obtaining state of affair that the world instantiates (now) being such that Cesar crossed the Rubicon. Given that such past-tensed properties are taken to be primitive and irreducible, it is plausible to maintain that in this view present is constituted (in our intended sense) by past-tensed states of affair. This is, of course, compatible with our claim that the present is not constituted by future-tensed states of affairs. Indeed, the Lucretian usually does not postulate futuretensed primitive properties along with the past-tensed ones, precisely in order to respect the asymmetry intuition (see also [45]). However, a perverse interpretation of Lucretianism, in which also irreducible future-tensed properties are at present instantiated by the world would be a trouble-maker for us. We set aside this worry for the purpose of the paper.

8 Does this come with failure of bivalence? Again, not necessarily, but we can assume that bivalence fails to keep things simple. 
We can make more rigorous the notion of weak indeterminate present by appealing to two roles times can play in claims concerning the obtaining of states of affairs, which we will refer to through the expressions "at t" and "as of t", respectively. Intuitively, if a state of affairs obtain at $t$ then $t$ is the temporal position of its obtainment, and if as of $t^{*}$ a state of affairs obtain at $t, t^{*}$ contains "all that it takes" for the state of affairs to obtain at $t$. We then take the first notion as a primitive that can be characterised as in (at $t$ ) below, while the second notion is explicitly defined in (as of $t$ ) below.

(at $t$ ) If a state of affairs $\mathrm{F}$ obtains/fails to obtain at $t$, then the proposition $\mathrm{P}$ that expresses $\mathrm{F}$ is true/false at $t$. If it is indeterminate whether $\mathrm{F}$ obtains at $t$, then $\mathrm{P}$ is unsettled at $t$

(as of $t$ ) As of $t$, a state of affairs $\mathrm{F}$ obtains/fails to obtain/is such that it is indeterminate whether it obtains at $t^{*}$ if and only if the states of affairs that constitute $t$ entail/fail to entail/neither entail nor fail to entail that $\mathrm{F}$ obtains at $t^{* 9}$

If, along with state of affairs talk, we also allow for the notion of a state of affairs' internal negation, indeterminacy with respect to the obtainment of $\mathrm{F}$ can be construed as being indeterminate which of $\mathrm{F}$ and its internal negation not-F obtains. Thus, if it is unsettled whether there will be a space battle around Terok Nor in 400 years, it is undetermined which of the two states of affairs [there will be a space battle around Terok Nor in 400 years] and [there will not be a space battle around Terok Nor in 400 years] obtains.

A weak indeterminate present (WIP) is a time that is constituted only by determinate states of affairs, but for which it is indeterminate which contingent future-tensed states of affairs obtain, namely which between some futuretensed states of affairs and their internal negations obtain. By resorting to metrical tense operators ("it will $n_{n}$ be the case that" to be read as "it will be the case in $n$ units of time that"), we can put it schematically as follows.

WIP A time $t$ is a WIP iff $t$ is present, and as of $t$ it is determinate which state of affairs constitute $t$, and for some present tensed proposition $\phi$ and positive number $\mathrm{n}$ it is indeterminate which of the future-tense state of affairs [it will $\mathrm{n}_{\mathrm{n}}$ be the case that $\phi$ ] or its internal negation [it will $\mathrm{n}$ be the case that not- $\phi]$ obtains at $t^{10}$

9 The two roles are analogous to the double indexing for evaluation of tensed propositions in the relativistic semantics of [34] (the idea is already in [46].). Roughly, "at $t$ " corresponds to the time of utterance, and "as of $t$ " to the time of assessment. However, the notion used in the existing literature are entirely semantic in nature: they are specification of the evaluation of propositions. The notion that we make use of here have an explicit metaphysic import: they are specification of the obtainment of states of affairs. Note that the semantic notion of truth is used only in the characterization of the primitive notion expressed by "at $t$ ", and the notion of entailment in (as of $t$ ) is a relation between two states of affair.

10 Note that the definition of WIP is B-theoretically acceptable. Although it does contain the predicate "present", nothing in what we say here forces us to read it in a metaphysically substantive way. It can be read simply as an indexical expression. The same will go for SIP. 
The received view explains the openness of the future via the weak indeterminate present. If it is unsettled whether there will be a battle around Terok Nor in four hundred years, then as of now, it is indeterminate whether the state of affairs that [there is a space battle around Terok Nor] or the state of affairs that [there is no space battle around Terok Nor] obtains in 2420. But when 2420 comes, it will no longer be indeterminate which state of affairs obtain in 2420. More generally, for any time $t$, some time $t^{*}\left(\right.$ with $t^{*}>t$ ), some positive number $n$, and every proposition of the form $<$ It will be the case that $\phi>$ (where $\phi$ is present tense, and does not contain hidden references to the future) that is unsettled at $t$ :

$\left(\mathbf{i}_{\mathbf{w}}\right)$ As of $t$, it is indeterminate which of the two future-tensed state of affairs [it will $l_{n}$ be the case that $\left.\phi\right]$ and $\left[\right.$ it will $_{n}$ be the case that not- $\phi$ ] obtains at $t$

$\left(\mathbf{i i}_{\mathbf{w}}\right)$ As of $t$, it is indeterminate whether $[\phi]$ obtains or [not- $\left.\phi\right]$ obtains at $t^{*}$ $\left(\right.$ iii $\left._{\mathbf{w}}\right)$ As of $t^{*}$, it is determinate which between the two future-tensed state of affairs [it will ${ }_{n}$ be the case that $\phi$ ] and [it will ${ }_{n}$ be the case that not- $\phi$ ] obtains at $t$

$\left(\mathbf{i v}_{\mathbf{w}}\right)$ As of $t^{*}$, it is determinate whether $[\phi]$ obtains or [not- $\left.\phi\right]$ obtains at $t^{*}$

We can now pinpoint more precisely the received view as the thesis that the locus of indeterminacy is the future insofar as it is still future. The future is open because there is indeterminacy with respect to what future-tensed state of affairs presently obtain, and such indeterminacy in the present is in turn (given that the present is otherwise constituted only by determinate state of affairs) explained by the indeterminacy with respect to which "corresponding" present-tensed states of affairs will obtain. More schematically, the received view can be construed as WIP-Openenness below.

(WIP-Openness) The future is open if and only if for any times $t$ and some time $t^{*}\left(\right.$ with $\left.t^{*}>t\right)$, and for some $\phi,\left(\mathrm{i}_{\mathrm{w}}\right)$ to $\left(\mathrm{iv}_{\mathrm{w}}\right)$ holds, and $\left(\mathrm{i}_{\mathrm{w}}\right)$ because $\left(\mathrm{ii}_{\mathrm{w}}\right)$

For instance, assume that as of $t$, there is indeterminacy with respect to which future-tensed state of affairs obtains at $t$, namely whether [it will be the case in four hundred years that there is a space battle around Terok Nor] or [it will be the case in four hundred years that there is no space battle around Terok Nor] obtain. Therefore, the present time is indeterminate, but only because as of the present there is indeterminacy with respect to what states of affairs constitute future times. But when future times in turn become present, the indeterminacy will be resolved: there is no indeterminacy as of at $t^{*}$ with respect to which one of the two (mutually exclusive) states of affairs [there is a space battle around Terok Nor] and [there is no space battle around Terok Nor] obtains at $t^{*}$. Finally, as we should expect, given that what holds for space battle in the future holds in general for contingent matters, it is easy to see that WIP-Openness complies with the adequacy condition AC that the truth value of future contingents is unsettled. 


\section{Weak versus Strong Indeterminate Present}

Although consistent, WIP-Openness is somewhat unstable. It explains the openness of the future in terms of the present being weakly indeterminate and the weak indeterminacy of the present in terms of the indeterminacy of the future relative to the present. One may think that this is how things should be: future times are open only until they are future. The locus of the indeterminacy that grounds the openness of the future can be neither in the present per se, nor in the future per se, but it must lie somewhere "in the middle". We do not mean to provide any knock-down argument against such a train of thoughts. However, we believe that once we assume that the aim is to model a metaphysically robust idea of openness, the received view is unsatisfactory. Even if there is indeterminacy now with respect to which present-tensed states of affairs will obtain, given that the indeterminacy will resolve, there is a mapping between the propositions that are unsettled as of a time $t$ - the future contingents - and certain corresponding state of affairs that will be determinate when the future arrives. But then, what is it in reality that makes the relation between the present and the future the locus of indeterminacy, given that they are both constituted only by determinate state of affairs? It is very tempting to reply that what makes the relation between the present and the future an indeterminate matter is just an epistemic factor. From the point of view of the present we can't see precisely, as it were, what the future is like. In other words, one might think that if all the states of affairs are determinate, both those in the present and those in the future, then the weak indeterminacy in the present could be explained away epistemically.

There are several reactions one can have to this criticism of WIP-Openness, assuming it is on the right track. One can insist that if we take on board some further metaphysical assumptions, such as the lack of existence of the future (cf. presentism or growing block), or a substantive notion of passage as a shift from the indeterminate to the determinate, the objection is blunted. As we have made clear, though, we are interested in what is shared among many views about the openness of the future. Insofar as this rejoinder is based on auxiliary substantive thesis, which someone who accepts WIP-Openness can reject (stick to the two examples above: a B-theorist can accept neither), then we will simply discard it. ${ }^{11}$

\footnotetext{
11 As a reviewer has pointed out, it is not clear at all that our own proposal is more parsimonious. We agree that the issue is open. However, the reason we discard this option is that it fails to comply with the requirement to be neutral with respect to various metaphysics of time. A reason that is entirely internal to the dialectics of the paper: we are setting on a side this option because it does not concerns versions of WIP that we are considering here. We are not denying that a "metaphysically loaded" version of WIP may turn out to be the best account overall. (Incidentally, as we will see, our own proposal is neutral at least with respect to the A-theory vs. B-theory debate.)
} 
Another reaction is to revise the received view by inverting the order of explanation and claim that it is indeterminate what the future is like because the present is indeterminate, as in WIP-Openness-revisited below. ${ }^{12}$

(WIP-Openness-revisited) The future is open if and only if for any times $t$ and some time $t^{*}$ (with $\left.t^{*}>t\right)$, and for some $\phi,\left(\mathrm{i}_{\mathrm{w}}\right)$ to $\left(\mathrm{iv}_{\mathrm{w}}\right)$ holds, and $\left(\mathrm{ii}_{\mathrm{w}}\right)$ because $\left(\mathrm{i}_{\mathrm{w}}\right)$

If things stand as the WIP-Openness-revisited has them, the future can be said to be the locus of indeterminacy (to be indeterminate with respect to which present-tensed states of affairs obtain) only in a derivative way, as a consequence of being indeterminate which future-tensed state of affairs obtain in the present. An instability very similar to the one pointed out for the WIPOpenness shows up in the revised version too: the openness of the future is explained in terms of the present being weakly indeterminate, but the indetermination of the present concern only future-tensed state of affairs. Indeed, the suspicion is that it may be historically indeterminate which one of WIPOpenness and the WIP-Openness-revisited is the received view. But more to the point, insofar as the only notion of indeterminacy we have at our disposal is the WIP, the openness of the future looks very much like a brute fact. As of itself, no time is constituted by indeterminate state of affairs, the indeterminacy of the future is only from the point of view of the present, and this relation between the present and the future - as suggested - is more naturally construed in epistemic terms, rather than as a feature of reality, given that both the present and the future, as of themselves are fully determined.

In this section, we will explore an alternative in which the indeterminacy of the future is explained in terms of a more robust idea of indeterminacy of the present. The hypothesis is that the present is partly constituted by state of affairs that entail indeterminacy about the future. Those present state of affairs ground the indeterminacy concerning the constitution of future times, and thus also the indeterminacy concerning which future-tensed state of affairs obtains in at the present time.

Let us start by considering what strong indeterminacy in the present consists in. In the contemporary debate, we find two main ways to characterise metaphysical indeterminacy. The divide is over whether indeterminacy has to be captured at the level of state of affairs, or at the level of objects. In the former case (see e.g. [4]), it is sometimes indeterminate that a certain state of affairs A obtains, for instance because it is neither determinate that A obtains, nor that non-A obtains, while in the latter case (e.g. [50]) sometimes an indeterminate state of affairs (determinately) obtains, for instance because a certain object $x$ instantiate a certain determinable property (being coloured, say), but fail to instantiate any of its determinate (being red, green, etc.). The

\footnotetext{
12 Of course, one could also react by concluding that the future is not open after all, and that its indeterminacy has no metaphysical source, being just a matter of ignorance. One of the two authors of the paper is indeed very sympathetic to this conclusion, and see the present paper as an exploration of an alternative.
} 
weak indeterminate present is indeterminacy as to which state of affairs obtain, it is thus of the first type. As for the strong indeterminate present, both options seem viable: the future may be open in virtue of it being indeterminate which present-tensed states of affairs presently obtain, or it may be so because some objects presently fail to determinately instantiate a property.

We will opt for the object level conception of indeterminacy, because better suits the quantum model developed at the end of the paper, although we will not argue for this choice here on independent grounds. Indeterminacy of presently obtaining present-tensed state of affairs is then to be understood as the obtainment of an indeterminate state of affairs - and not as whether a determinate state of affairs or its internal negation obtains.

How are we to characterise an indeterminate state of affairs? Although we will pass over some of the details here, it is useful to provide some characterisation. We will piggyback on the account in [50] to give an idea:

(Wilson-style Indeterminate State of Affairs) A state of affairs is (Wilsonstyle) indeterminate iff a certain object instantiates a determinable property, but more than one determinate of that determinable ${ }^{13}$

An indeterminate state of affairs, on this view, occurs if the relationship between an object and its determinate and determinable properties is of a certain type. Therefore, the indeterminacy "at the object level" is nonetheless individuated by looking at the structure of the indeterminate states of affairs. We can now provide a characterisation of the strong indeterminate present (SIP).

(SIP) A time $t$ is a SIP iff $t$ is present and $t$ is constituted by at least one indeterminate state of affairs

In order for SIP to provide an explanation of the unsettledness of future-tensed propositions, it should comply with the following adequacy condition.

(AC-SIP) : for any times $t$ and some time $t^{*}$ (with $t^{*}>t$ ) and some $\phi$, if $t$ is a SIP, then as of $t$, it is indeterminate whether $[\phi]$ obtains or [not- $\phi]$ obtains at $t^{*}$, and it is so because of some indeterminate state of affairs that constitutes $t$

Why should one think that robust metaphysical indeterminacy in the present can explain the indeterminacy that characterise the future? Think again of the example of the protest that we given in the first section. It is because the members of the Congress and the protesters have indeterminate mental contents that it is still open whether there will be a protest tomorrow or not. To simplify the example, consider Senator Robert. At noon, he is still utterly undecided whether he will vote yes or no in the 18:00 voting. As of

\footnotetext{
13 This is the "glutty" version of indeterminacy. Wilson allows also for a "gappy" version in which no determinate of that determinable is instantiated. Since we believe there are good reasons, coming from quantum mechanics, for preferring the "glutty" approach (see $[13])$, this will be our only focus in this paper.
} 
noon, it is thus undetermined whether at 18:00 he will vote yes or no. The relevant state of affairs about Senator Robert that constitute noon here can be thought of as composed by a determinable (e.g., being in a mental state about tonight voting) and more than one determinate of that determinate (e.g., intending to vote yes, intending to vote no). Those states of affairs ground the present indeterminacy of the 18:00 voting with respect to Robert's behaviour. Of course, the example does not cut much metaphysical ice, since the kind of indeterminacy at issue here is clearly mere representational indeterminacy (we do not need to posit an indeterminate reality in order to have indeterminate thoughts). What matters here is to give an idea of the structural connection between the indeterminate states of affairs that constitute the present and the state of affairs with respect to which the future is indeterminate.

In general, we can say that state of affairs have as constituents individuals $\left(s_{1}, s_{2}, \ldots\right)$ exemplifying properties. ${ }^{14}$ Those properties are determinable properties $\left(D_{1}, D_{2}, \ldots\right)$, and each determinable property has its own corresponding pool of determinate properties $\left(A_{D}, B_{D}, \ldots\right)$. In order to allow for indeterminate state of affairs as characterized above, we can introduce also complex properties, whose constituents are other properties. A complex property constituted by properties $A$ and $B$, for instance, will be labelled $A / B$. Importantly, we also need to allow that some complex properties are composed by determinates of the same determinable. For instance, $A_{D} / B_{D}$ is a complex property composed by two determinate properties of the determinable $D$. In line with Wilson's idea introduced before, we can say that when a state of affairs exemplifies a determinable property $D$ and some such complex property constituted only by $D$ 's determinate property is an indeterminate state of affairs. What interests us is to express the relation between a present state of affairs $\left[s, D, A_{D} / B_{D}\right]$ that is constituted by a system $s$, a determinable $D$, and some complex property $A_{D} / B_{D}$ and the state of affairs $\left[s, D, A_{D}\right]$ and $\left[s, D, B_{D}\right]$ concerning the same system s and determinable $D$, but only one of the determinate property $A_{D}$ or $B_{D}$. Such a relation can be expressed by the following four clauses. For any time $t$ and some time $t^{*}$ (with $t^{*}>t$ ) and some individual $s$, determinable $D$, and some of $D$ 's determinate $A_{D}$ and $B_{D}$ :

(i $\left.\mathbf{i}_{\mathbf{s}}\right)$ As of $t$, an indeterminate state of affairs $\left[s, D, A_{D} / B_{D}\right]$ obtain at $t$

(ii $\left.\mathbf{s}_{\mathbf{s}}\right)$ As of $t$, it is indeterminate whether $\left[s, D, A_{D}\right]$ obtains or $\left[s, D, B_{D}\right]$ obtains at $t^{*}$

(iii s $_{\text {) }}$ As of $t^{*}$, it is determinate which between the two future tensed state of affairs $\left[\right.$ it will $\mathrm{n}$ be the case that $\left.\left[s, D, A_{D}\right]\right]$ and $[$ it will $\mathrm{n}$ be the case that $\left.\left[s, D, B_{D}\right]\right]$ obtains at $t$

$\left(\mathbf{i v}_{\mathbf{s}}\right)$ As of $t^{*}$, it is determinate whether $\left[s, D, A_{D}\right]$ obtains or $\left[s, D, B_{D}\right]$ obtains at $t^{*}$

\footnotetext{
14 This characterization may remind of [28] theory of events. However, note that here times are not constituent of state of affairs/facts. Rather, state of affairs obtain at times. In other terms, we are dealing here with tensed state of affairs.
} 
According to what we call the SIP-Openness view, the indeterminacy with respect to what states of affairs constitute the future is explained in terms of a strong indeterminate present.

(SIP-Openness) The future is open if and only if for any time $t$ and some time $t^{*}$ (with $t^{*}>t$ ) and some individual $\mathrm{s}$, determinable $D$, and some of $D$ 's determinate $A_{D}$ and $B_{D}$ : $\left(\mathrm{i}_{\mathrm{s}}\right)$ to $\left(\mathrm{iv}_{\mathrm{s}}\right)$ holds, and $\left(\mathrm{ii}_{\mathrm{s}}\right)$ because $\left(\mathrm{i}_{\mathrm{s}}\right)$

SIP-Openness compiles with AC-SIP. It is also easy to see how it can be used to account for the unsettledness of future contingents and so how it complies with $\mathrm{AC}$ too, once we accept the following plausible principle Link.

(Link) If as of a time $t$ there is indeterminacy with respect to which presenttensed states of affairs $F_{1}, F_{2}, \ldots$ obtain at a future time $t^{*}$, then as of $\mathrm{t}$ there is indeterminacy with respect to which future-tensed versions (it will be the case that $F_{1}$, it will be the case $F_{2}$, etc.) of the state of affairs $F_{1}$, $F_{2}, \ldots$ obtain at $t$

Given Link, SIP-Openness entails that if a time is a SIP, then it is also a WIP (as we should expect). Given (at t), SIP-Openness entails the unsettledness of the future contingents. Note, however, that by appealing to SIP-Openness rather than the WIP-Openness (or WIP-Openness-revisited), we can bottom out the explanation of the unsettledness of the future contingent in a "stable" form of metaphysical indeterminacy: the determinate present obtainment of an indeterminate state of affairs. In so far as it is better to avoid both to be unstable with respect to the source of indeterminacy, and to be too similar to an epistemic form of indeterminacy, SIP-Openness is preferable to the received view (either as WIP-Openness or WIP-Openness-revised). ${ }^{15}$

SIP-Openness tells us something about the internal structure of present indeterminate states of affairs, and their connection with the structure of the state of affairs for which it is indeterminate whether they will obtain or not. Of course, SIP-Openness does not tell us why indeterminate state of affairs in the present can explain indeterminacy as of now of what state of affairs will obtain, it just gives us an idea of the structural connection between those state of affairs that is required by such an explanation. To answer this question we need to put more metaphysical flesh on the bones of SIP-Openness, by introducing a background theory to tell us what $s, D, A_{D}, B_{D}$, and $A_{D} / B_{D}$ are.

In the next section, we will introduce the basics of the spontaneous collapse approach to quantum mechanics, which will then be used, in section (5), as a background theory for SIP-Openness.

\footnotetext{
15 Note that SIP-Openness is perfectly compatible with a form of B-theory. And indeed it is formulated in a tenseless language. It is true that SIP (as much as WIP) contains the predicate "present", but as already noted, nothing hinges on reading it in a metaphysically robust sense.
} 


\section{The Indeterminate Present in Spontaneous Collapse Interpretations of QM}

In order for the SIP-Openness model to be true in our world, we need the underlying theory to meet two conditions. First, we need the theory to possess the resources to distinguish between determinate and indeterminate state of affairs, roughly in the way described in section 4 above, and to entail the existence of the latter. Second, the theory we are looking for has to provide instances of the schema given by SIP-Openness. In this section we shall focus on the former of these conditions, and then move to the latter on section 6 .

The idea that quantum mechanics displays a deeper sense of indeterminacy has been discussed in the physics literature since the very birth of the theory. However, it is only in the past few years that philosophers have started to pay serious attention to this phenomenon and to its various conceptual consequences. Many authors (cf. [19], [10], [49], [13], inter alia; for criticisms, see [21]) have reflected on the pervasiveness of a feature known as lack of value definiteness (henceforth, LVD). LVD is indeed largely independent of how we interpret quantum mechanics, although different interpretations will account for it differently.

Let us consider a simple example. If we know, say, that the electron $e$ has spin-down on the $x$-axis (suppose because we measured it), we thereby also know that its spin on the other two axes cannot in principle be assigned. We then have the following states:

(a) $e=|\downarrow x\rangle$

(b) $e=|\downarrow x\rangle=\frac{1}{\sqrt{2}}|\downarrow z\rangle+\frac{1}{\sqrt{2}}|\uparrow z\rangle$

(c) $e=|\downarrow x\rangle=\frac{1}{\sqrt{2}}|\downarrow y\rangle+\frac{1}{\sqrt{2}}|\uparrow y\rangle$

Where (a) means that the electron is in an eigenstate of having the value down for the observable $x$-spin, and (b) and (c) express the empirical fact that being in an eigenstate of the observable $x$-spin implies being in a superposition of the observables that are incompatible with $x$-spin, namely $z$-spin and $y$-spin respectively. Since to every eigenstate there are some corresponding superposition states, LVD is pervasive in standard quantum mechanics. More generally, let us consider the standard way of assigning properties to quantum systems, the Eigenstate-Eigenvalue Link (EEL) ${ }^{16}$ :

EEL A quantum system $s$ has a definite value $v$ for the observable $O$ iff it is in an eigenstate of $O$ having eigenvalue $v$

From EEL, we can derive that systems that are not in eigenstate of having value $v$ for the corresponding properties, do not possess a definite value for those properties. This is, in a nutshell, LVD in quantum mechanics. And the interpretative issues that behind EEL and LVD are the main reasons for proposing an account of quantum indeterminacy in the first place.

16 See [48] and [23] for a critique of the theoretical utility of EEL in standard QM. 
Now using the Calosi \& Wilson's account of QI (cf. [13]), through the EEL, we have that:

(1) $e$ has the definite value down for the property $x$-spin

(2) $e$ does not have a definite value for the properties $y$-spin and $z$-spin

Being instances of LVD, Calosi \& Wilson ([13]) suggest that we read the states corresponding to (2) as indeterminate states of affairs. Take, for example, the state corresponding to $y$-spin. We have an individual, the electron $e$, a determinable, the observable corresponding to $y$-spin, and the determinate of that determinable, down and up. Thus, at the time at which the state occurs, call it $t$, we have the following indeterminate SOA:

(3) $\left[e, y \text {-spin, down }{ }_{y-\text { spin }} / \text { up }_{y-s p i n}\right]^{17}$

Another major interpretative issue in quantum mechanics is that we do not directly experience superposition states like the above. Whenever we perform measurements, the system we look at "picks", so to speak, one of the superposed terms. In other words, after a measurement on $y$-spin, from (3) we get to one of the following:

(4) $\left[e, y\right.$-spin, down $\left.{ }_{y-s p i n}\right]$

(5) $\left[e, y\right.$-spin, up $\left.p_{y-s p i n}\right]$

Explaining why and how this happens is, in large part, the business of providing an interpretation to quantum mechanics. The orthodox view, first formulated by von Neumann, is that measurements induce the wavefunction that describes the dynamical evolution of quantum systems to collapse into eigenstates, thereby eliminating superpositions. It is not our aim here to discuss which approach to quantum mechanics is to be preferred. Rather, we want to point out that if (a) we interpret LVD as Calosi \& Wilson ([13]) suggest, and if (b) we assume that the collapse of the wavefunction can be described satisfactorily, then quantum mechanics might be seen as a candidate underlying theory for a model like SIP-Openness. As regards to (b), it is no secret that von Neumann's view on this issue has been criticized on many respects. Above all, the worry is that the notion of measurement is not well defined in theory. However, spontaneous collapse QM was introduced mainly in order to overcome such difficulties, and yet agreeing with von Neumann's view about the idea that the linearity of dynamics has to supplemented with a non-linear component.

The Spontaneous Collapse Models are a family of interpretations of Quantum Mechanics that were first introduced by Ghirardi, Rimini, and Weber ([22]; GRW henceforth), building upon suggestions made by [41], and [24]. As

\footnotetext{
17 This can be easily generalized. Take a system s and one of its observables $O$, with distinct eigenstates $|\psi\rangle$ and $|\phi\rangle$. Any linear combination $|\omega\rangle=\mathrm{a}|\psi\rangle+\mathrm{b}|\phi\rangle$ is a superposition. The resulting quantum state $|\omega\rangle=\mathrm{a}|\psi\rangle+\mathrm{b}|\phi\rangle$, obtaining at a certain time $t$, is then to be interpreted as $[s, O, \psi \mathrm{O} / \phi \mathrm{O}]$.
} 
with all other interpretations of QM, such as the Bohmian (cf. [9]), or the Everettian (cf. [20]), the main reason why GRW was first formulated was to address the measurement problem of quantum mechanics. Roughly, this problem comes from the assumption that the standard (uninterpreted) formalism of QM is universally valid. A crucial feature of this formalism is that the temporal evolution of quantum systems is governed by the Schrödinger's equation, which, among other features, is deterministic, and linear. The linearity of this equation allows the formalism to describe states of superposition. These states are practically impossible to visualize, and yet very easy to describe mathematically. To give an example, you can think of an electron e, and one of its properties (observables); for instance, its spin component along a certain axis. This property can be instantiated by the electron only in two ways: it can be either spin-up, or spin-down. When this happens, we say that the electron is in an eigenstate of the relevant observable (property), and we write $e=|\uparrow\rangle$ or $e=|\downarrow\rangle$, respectively. Now, the linearity of the Schrödinger's equation implies that, for every two solutions to the equation, the linear combination of them (their sum) is also a solution. Thus, we do not only have eigenstates, but also states of superposition of having, in the above example, both spin-up and spin-down: $e=|\uparrow\rangle+|\downarrow\rangle$. These states are pervasive in QM, given the existence of incompatible observables - spin components in mutually orthogonal directions are a famous example. Whenever a quantum system is an eigenstate of a certain observable, it will always be in a superposition of the observable that is incompatible with the one in the eigenstate. The measurement problem emerges from the simple consideration that, apparently, macroscopic objects are never in superposed states (cats are never both alive and dead at the same time). And therefore, as nicely summarized by Bell, "either the wavefunction, as given by the Schrödinger's equation, is not everything, or it is not right" ([5], p. ).

The idea of the collapse of the wavefunction would solve the measurement problem simply by breaking the Schrödinger's dynamics. According to von Neumann's collapse postulate, the dynamics breaks whenever a measurement is performed. Therefore, there are two different physical processes according to von Neumann: one is linear, deterministic, and described by the Schrödinger's equation; and the other one is stochastic, and it is described by the collapse postulate. Almost everyone agrees today that this proposal is untenable for at least two reasons: it does not explain what a measurement is, and it does not explain why measurement plays such a crucial role.

In order to address these issues, GRW proposes a realistic collapse model that is very close in spirit to von Neumann's proposal, but avoids its explanatory shortcomings. Instead of occurring through measurements, in GRW collapses occur spontaneously and are governed by a new law. This law is completely different from Schrödinger's equation: it is not linear, it is not deterministic, and it is not time reversal invariant. The collapse is explained by the assumption that every particle has a certain probability per unit time of undergoing a hit, and every hit makes the particle jump to a definite state. The wavefunction that describes the particle after a hit gets multiplied by a 
narrow Gaussian function stretched to infinity in both sides. The exact point on which the hit is localized within the Gaussian is determined randomly. However, given that we want to recover the statistics of standard QM, the probability distribution (within the Gaussian) is given by the square of the wavefunction amplitude before the hit, exactly as for the Born rule.

Another crucial feature of objective collapse interpretations of QM is worth mentioning. It is usually assumed that collapses occur in a preferred basisin GRW, for instance, it is position. The requirement of a preferred basis is necessary insofar as one wants to take collapses as real physical processes. The choice of position is usually justified by the fact that macroscopic superposition states seem the least acceptable.

GRW model and the like seem a promising way to go in order to solve the main conceptual difficulties of QM. However, it also has some drawbacks. For instance, it has been argued that the introduction of a new dynamical law is ad $h o c$, or that the model has certain problems with dimensionality and locality (see [30], [31]). It is not our aim here to address these issues, but there is one problem of GRW that is crucial for our arguments, so we will spend few words on it before moving to the next section.

Recall that, after collapse, the amplitude is not localized in a finite region, because the Gaussian function is stretched infinitely in all sides. This means that in GRW we never have collapses to position eigenstates, but only near eigenstates. This issue is known in the literature as the tails problem. If we require that a quantum object possess a determinate property if and only if it is in an eigenstate of the relevant observables (as with the Eigenstate-Eigenvalue link), it is easy to see how from GRW it would follow that objects never possess definite properties. In order to respond to this problem, proponents of GRW have tried to somehow relax EEL (cf. [2], [31]). For instance, one can admit that objects possess definite properties also when the relevant observable is near an eigenstate. This proposal is called the fuzzy link (cf. [2], [16]):

Fuzzy Link A system has a determinate value for a given property iff the squared projection of its state onto an eigenstate of the corresponding operator is greater than 1 - $P$, where the determinate value is the eigenvalue for that eigenstate

$P$ is just a parameter that can be chosen as expressing what counts as definite. The bigger it is, the less the state needs to be close to eigenness in order to count as possessing the property. Notice that the fuzzy link is not fuzzy as to whether or not a property is instantiated, rather, it is explicitly fuzzy as to what is the border (the choice of $P$ ).

Or, instead of the fuzzy link, one can say that objects instantiate properties vaguely, and with degrees that corresponds to the relevant probability. This proposal has been recently put forward by Lewis (cf. [31]):

Vague Link A system has a determinate value for a given property to the extent that the squared projection of its state onto an eigenstate of the 
corresponding operator is close to 1 , where the determinate value is the eigenvalue for that eigenstate

Here instantiation of properties does not come as all or nothing. For instance, my laptop instantiates the property of being on my desk now only to a certain degree, that can be calculated by considering the probabilities given by every Gaussian function associated with all the laptop's and desk's particles. And of course, whatever this probability is, it is enough close to 1 to allow us to say, for all practical purposes, that the laptop is definitely on the desk.

Each of the above proposals has drawbacks. As for the fuzzy link, what is allegedly the main problem is that it implies that properties sometimes behave as if they are not instantiated as the link says. For instance, according to the fuzzy link, an object possessing the property of being on my desk can sometimes behave as if it is on my carpet. Although this would happen with a very small probability, and for all practical purposes we can avoid countenancing this possibility, from a conceptual point of view this is highly problematic. The point is that it would imply the falsity of those theories according to which properties are individuated only by means of their dispositions. As for the vague link, it might seem odd to many to posit such a pervasive vagueness in the physical world. After all, from the vague link it follows that we are always wrong in attributing determinate properties to objects.

The tails problem is not only particularly pressing for anyone who attempts to interpret spontaneous collapse models as entailing metaphysical indeterminacy. It becomes even more pressing for those who wish to provide a theory of openness based on such an interpretation of QM. In his recent book, Callender (cf. [12]) seems to recognize this problem quite well. While in the business of rejecting the various attempts of arguing for the open future based on QM, Callender considers the possibility to argue that the superposition/eigenstate distinction maps directly the openness-of-future/fixity-of-the-past one. He then notices the following:

[...] the symmetry of Hilbert space implies that we can write out our wavefunction in any of an indefinite number of bases, e.g., position, momentum, spin. A wavefunction that is a superposition in one basis may not be a superposition in another; for instance, the wavefunction of $x$-spin down is a superposition of up and down spins in the z-spin direction. Here a collapse to fixity in $x$-spin buys openness in $z$-spin. ([12], p. 95)

As he himself recognizes, though, to this objection one can respond that in theories such as GRW, a collapse always occurs in a preferred basis - as we said above, in the position one - and that we should only care about position superpositions states and eigenstates when we speak about the asymmetry between fixed past and open future. However, Callender immediately rejoins a second objection by reminding us that "in any realistic collapse theory such as GRW one doesn't get collapses onto eigenstates, but only near eigenstates" ([12], p. 95). Hence, even granting, by focusing only on position states, that 
there is a mapping between superposition/eigenstate and open/fixed, in GRW collapses are never to eigenstates, but only near ones. And without eigenstates, the thought goes, we will not end up having openness properly.

It is clear from those considerations that the tails problem is central to the viability of the whole project of a spontaneous collapse model. If the problem cannot be solves, GRW has to be abandoned. However, as we have argued above, various solutions to the tails problem has been put forward, and Callender does not seem to argue against them in details. And since our model is already conditional on the truth of GRW, we are perfectly happy to conditionalise the model over the claim that the tails problem can be solved ${ }^{18}$.

\section{The Strong Indeterminate Present in QM}

Let us see now how spontaneous collapse models can be used to model SIPOpenness. In the case of (4) and (5) above, the two clauses $\left(\mathrm{i}_{\mathrm{s}}\right)$ and $\left(\mathrm{ii}_{\mathrm{s}}\right)$ introduced in section 4 are met as follows (and analogously for (iii $\left.i_{\mathrm{s}}\right)$ and $\left(\mathrm{iv}_{\mathrm{s}}\right)$ ).

$\left(\mathbf{i}_{\mathrm{s}}^{*}\right)$ As of $t$, an indeterminate state of affairs $\left[e, y\right.$-spin, down $\left.n_{y-s p i n} / u p_{y-s p i n}\right]$ obtains at $t$

\footnotetext{
18 Two anonymous reviewers of this journal protest that conditionalising on the possibility that the Tails Problem could be solved is not correct in this context, and that we should rather conditionalise on some specific solution. We believe that the source of the misunderstanding here relates to what exactly the Tails Problem is, and to what it is supposed to show. It is an overall assumption of any realistic collapse theory like GRW that measurement outcomes are definite - the pointer is always either up or down. To better see this, take for instance Maudlin's ([36]) well-known trilemma regarding the measurement problem. According to Maudlin, we can solve the problem by dropping (at least one) of the following three tenets of standard QM: (a) completeness, (b) unitary evolution, (c) definiteness of outcomes. Now, collapse theories drop (b), thus maintaining the determinacy of outcomes (contrary to Everettian-type of interpretation, which drops it). This means that in any such theory we do have determinate SOAs basically by assumption. The tails problem do not relate to the fact that outcomes are not determinate, but rather relates to how it could happen that this is the case, given that the dynamical evolution never evolves into eigenstates. We do not mean to underestimate this latter problem; if it cannot to be solved (as recently argued, for instance, in [38]) then GRW would have to be abandoned. But the point is that the reason why it would is precisely that it cannot explain the definiteness of outcomes, which again is an assumption of these models. This arguably shows that no matter how we solve the Tails Problem (if we can solve it), the resulting ontology given by theories like GRW would be one where there exist determinate SOAs. As one of the reviewer points out, Peter Lewis' Vague Link [31] might be an important exception to the above line of reasoning. In effect, Lewis explicitly allows for indeterminacy in GRW also at the level of the post-collapse ontology. We grant that such a solution to the tails problem would be problematic for our view. However, we shall notice that it is not so clear whether, even adopting Lewis' Vague Link, a view close to ours could not be defended. Perhaps, for instance, it could be argued that the post-collapse states being sufficiently determinate - possessing the relevant determinate instantiated with a sufficiently high degree - is enough not merely from a pragmatic point of view, but at a metaphysical level as well. We take it to be an interesting issue whether such an extension of the model could be construed, but have to leave the details to another occasion. We thank both reviewers for inviting us to be clear regarding our take on this issue.
} 
$\left(\mathbf{i i}_{\mathbf{s}}^{*}\right)$ As of $t$, it is indeterminate whether $\left[e, y\right.$-spin,down $\left.n_{y-s p i n}\right]$ or $[e, y$ spin, $u p_{y-s p i n}$ ] obtains at $t^{*}$

Suppose a measurement occurs at $t^{*}$ and instantaneously collapses the wavefunction into one of the superposed terms. This means that, as of time $t$ before the collapse, an indeterminate state of affairs obtains (the superposition), and it is indeterminate which term of the superposition will obtain at $t^{*}$ after the collapse.

Recall now, that SIP-Openness is meant to provide an explanatory schema. It is not enough, then, that the two clauses are satisfied-we also need a motivation for believing that $\left(\mathrm{ii}_{\mathrm{s}}^{*}\right)$ happens because $\left(\mathrm{i}_{\mathrm{s}}^{*}\right)$ does $^{19}$. Our suggestion is that the notion of collapse does indeed play the required explanatory role. What is crucial here is that collapse is an intrinsically temporal notionwe cannot make sense of collapse without distinguishing between the time before its occurrence, and the time afterwards. This idea is not a radically new one. Many physicists seem sympathetic with the view we are defending here, according to which the collapse mechanism is apt to explain openness (cf. [32], [15], [25]). Here is, for instance, Lucas:

There is a worldwide tide of actualization - collapse into eigennessconstituting a preferred foliation by hyperplanes (not necessarily flat) of co-presentness sweeping through the universe - a tide which determines an absolute present [...] Quantum mechanics [...] not only insists on the arrow being kept in time, but distinguishes a present as the boundary between an alterable future and an unalterable past ([32], p. 10).

\section{And here is Gisin:}

Admittedly, time is a complex notion, or series of notions with many facets, time may be relative, difficult to grasp, etc. But time exists. Moreover, time passes. With spontaneous collapse theories, time exists and passes, the world out there exists and undergoes a stochastic evolution ([25], p. 7).

\footnotetext{
19 A reviewer of this journal raises a very interesting worry. It is a feature of GRW that whenever a certain particle undergoes a collapse, its wave function is immediately, once again, spread across space. This entails that to every evolution from indeterminacy to determinacy (to every collapse) there corresponds a somewhat symmetric evolution from determinacy to indeterminacy (the spreading of the wave function). Thus, the reviewer contends, one could well imagine a model similar to ours (by carefully rewriting the (i)-(iv) clauses) and argue that on GRW the past is open and the future fixed. Of course, this would be an unwelcome consequence. Notice, however, that for such an Open Past thesis to be true we would need an explanatory claim similar to our SIP-Openness, on which determinacy in the present explains indeterminacy in the past. We take it to be quite natural to reject that such a claim is a good explanation. Furthermore, the above worry seems to rest on the idea that GRW does not prefer one direction over the other. However, it is important to remind that in GRW the fundamental laws of dynamical evolution are temporally asymmetric - contrary to what happens with almost every other interpretation of QM. As a matter of fact, it has been even suggested that on GRW we can explain the temporal asymmetry of thermodynamics, along with the arrow of time given by direction of entropy (see [1]). Therefore, in GRW the indeterminacy-to-determinacy evolution is in fact priviledged.
} 
If we take the temporal component of collapse at face value, we shall conclude that what the notion does is to explain how physical systems change through time. It is natural, then, to take instances of $\left(\mathrm{ii}_{\mathrm{s}}^{*}\right)$ as explained (at least in part) by instances of $\left(\mathrm{i}_{\mathrm{s}}^{*}\right)$ : it is the fact that there is a superposition that explains why a collapse will occur. ${ }^{20}$

The resulting metaphysical picture is in line with the Strong Open Present hypothesis discussed in the first part of this paper. The locus of future unsettledness is the intrinsic indeterminacy that certain present states of affairs display. Superposed states are inherently "unstable", and tend to evolve into one of the open "options" that inhabit their future.

\section{Conclusions}

A different metaphysical explanation of the openness of the future can be given by considering what we call the strong indeterminate present. We have given a precise characterization of how this model works, and how it explains the openness of the future. Finally, by looking at quantum mechanics, we have provided reasons to believe that this model might apply to our own world, if a certain interpretation of QM is the correct one. Standard metaphysical explanations of openness are usually silent about the physical description of the underlying phenomena. Openness understood in terms of the collapse of the wavefunction provides a radically new, naturalistic account of openness. This is, we believe, an interesting result per se. Whether or not a similar model is true of our world, crucially will depend on whether or not the underlying physical theory (upon which we conditionalise) is itself true or not.

\footnotetext{
20 As a reviewer of this journal has pointed out, since collapses are extremely unlikely to occur for individual systems, it could be argued that clause (iv $\mathrm{s}_{\mathrm{S}}^{*}$ ) is not always met-as of $\mathrm{t}^{*}$, for the individual system that does not collapse, there is no determinate state of affairs that obtains. Although this is correct, we do not take it as an objection to the model. As a matter of fact the universe we live in is made of a large number of highly entangled particles. This means that for the vast majority of particles collapses are way more likely to occur than not. One could push this point further, and imagine a case of a perfectly isolated electron in an otherwise highly entangled universe. However, even in this case, it seems natural to assume that in such a universe the model will apply, given that it applies to a vast portion of the particles and in any case to the universe as a whole (that is considered as a system). In a way, we would imagine these entangled particles to drag forward passage of time for the isolated particle too. The reviewer acknowledges that this is correct, but then wonders whether this would entail that evidence for the open future depends on the existence of a large number of entangled particles. Our reply to this further worry is that it does, but rather than take this feature as a weakness of the model, we actually believe it is one of its main strengths. Suppose we take a universe populated by very few particles which never interacts one to another; would the future be open to them? We have to bite the bullet here, and agree that according to our model the future would not be open in that universe. However, we take our model to be motivated by a certain empirical theory which happens to be true in a universe with a large number of entangled particles, namely the actual universe. Although we understand that not everyone will be happy with such a reply, we nonetheless stress that the above objection is not a fatal one, but only shows one of the conceptual features of the model. For this reason we thank once again the reviewer for inviting us to clarify this issue.
} 
Acknowledgements We would like to thank Craig Callender, Peter Lewis, Jessica Wilson, and especially Claudio Calosi, and Jonathan Tallant for useful comments on previous versions of this manuscript. We also thank the audiences of the $\mathrm{V}$ and VI International Association for Philosophy of Time Annual Meeting in Seoul and Boulder (CO) for comments and suggestions. Finally, we would like to thank all the members of the Centre for Philosophy of Time in Milan and the Centre for Time in Sydney for useful discussion on a previous draft of the paper.

\section{Conflict of interest}

The authors declare that they have no conflict of interest.

\section{References}

1. Albert, D. Z., Quantum Mechanics and Experience, Cambridge, MA: Harvard University Press. (1992)

2. Albert D. \& Loewer B., "Tails of Schrödingers Cat", in R. Clifton (ed.), Perspectives on Quantum Reality, 81-92. Dordrecht, Springer (1996)

3. Barnes E. \& Cameron R., "The Open Future: Bivalence, Determinism and Ontology", Philosophical Studies, 146, 291-309 (2009)

4. Barnes E. \& Williams J. R. G., A Theory of Metaphysical Indeterminacy, in Bennett, K., \& Zimmerman, D. W., (eds.), Oxford Studies in Metaphysics, volume 6, 103?148. Oxford, Oxford University Press (2011)

5. Bell J. S., Speakable and Unspeakable in Quantum Theory. Cambridge, Cambridge University Press (1987)

6. Belnap N., Perloff, M., \& Xu, M., Facing the future, Oxford: Oxford University Press (2001)

7. Bernecker, S., \& Michaelian K., The Routledge Handbook of Philosophy of Memory, Routledge (2011)

8. Bigelow J. "Presentism and Properties," Philosophical Perspectives 10 (Metaphysics), 35-52 (1996)

9. Bohm D. "A suggested interpretation of the quantum theory in terms of hidden variables. I \& II," Physical Review 85. 166, ibid., 85, 180 (1952)

10. Bokulich A. "Metaphysical indeterminacy, properties, and quantum theory?," Res Philosophica, 91, 449-475 (2014)

11. Borghini A., Torrengo G, "The Metaphysics of the Thin Red Line," in F. Correia, A. Iacona (eds.), Around the Tree, Berlin, Synthese Library, Springer Verlag, 105-125 (2013)

12. Callender C. What Makes Time Special?, Oxford, Oxford University Press

13. Calosi C. \& Wilson J. "Quantum Metaphysical Indeterminacy?," Philosophical Studies, 176:1-29 (2018)

14. Cameron R., The Moving Spotlight. An Essay on Time and Ontology, Oxford, Oxford University Press (2015)

15. Christian J. "Passage of Time in a Planck Scale Rooted Local Inertial Structure," International Journal of Modern Physics D, 13:1037-1071 (2004)

16. Clifton R. \& Monton B. "Losing your marbles in wavefunction collapse theories?," British Journal for the Philosophy of Science, 50, 697-717 (1999)

17. Correia F., \& Rosenkranz S., Nothing to Come. A defence of the Growing Block theory of Time, Springer (2019)

18. Darby G. "Quantum Mechanics and Metaphysical Indeterminacy?," Australasian Journal of Philosophy 88 (2), 227-245 (2010)

19. Darby G. "Vague Objects in Quantum Mechanics," in Akiba, K. \& Abasnezhad, A., (eds.), Vague Objects and Vague Identity. New Essays on Ontic Vagueness, New York: Springer, 69-108 (2014)

20. Everett H. The Theory of the Universal Wave Function (1956), first printed in DeWitt and Graham (1973), 3-140. Reprinted in Barrett and Byrne (2012) 72-172 
21. Glick D. "Against Quantum Indeterminacy?," Thought, 6(3),204-213 (2018)

22. Ghirardi G. C., Rimini A., \& Weber T. "Unified Dynamics for Microscopic and Macroscopic Systems", Physical Review D, 34, 440-91 (1986)

23. Gilton M.J.R. Whence the eigenstate-eigenvalue link? Studies In History and Philosophy of Science Part B Studies In History and Philosophy of Modern Physics 55 (2016)

24. Gisin N. "Quantum measurements and stochastic processes?," Physical Review Letters, 52: 1657, and Reply, ibid, 53, 1776 (1983)

25. Gisin N. "Collapse. What Else?" (manuscript)

26. Iacona A., "Future Contingents and Aristotle's Fantasy," Critica 39 (117), 45-60 (2007)

27. Ingram D. \& Tallant J., "Presentism", Stanford Encyclopedia of Philosophy (2018)

28. Kim, J.Supervenience and Mind: Selected Philosophical Essays, New York, Cambridge University Press (1993)

29. Le Poidevin R. Change, Cause and Contradiction: A Defence of the Tenseless Theory of Time, London, Macmillan (1991)

30. Lewis P. J. "Four strategies for dealing with the counting anomaly in spontaneous collapse theories of quantum mechanics," International Studies in Philosophy of Science, 17, 137-142 (2003)

31. Lewis P. J. Quantum Ontology. A Guide to the Metaphysics of Quantum Mechanics, Oxford, Oxford University Press (2016)

32. Lucas J. R. "Transcendental tense II," Aristotelian Society Supplementary Volume, 72, 29-43 (1998)

33. Łukasiewicz, J. "Philosophical Remarks on Many-valued Systems of Propositional Logic." Polish Logic 1920-1939.Ed. Storrs McCall. Oxford: Clarendon, 40-65 (1967)

34. MacFarlane J. "Future contingents and relative truth," Philosophical Quarterly, 53(212): 321-336 (2003)

35. Markosian N., "The open past", Philosophical Studies 79, 1: 95-105 (1995)

36. Maudlin T. "Three measurement problems", Topoi 14, 7-15. (1995)

37. McCall S. A Model of the Universe, Oxford, Oxford University Press (1994)

38. McQueen, K. J., "Four tails problems for dynamical collapse theories", Studies in History and Philosophy of Modern Physics, 49, 10-18 (2015)

39. Miller K., "Time Travel and the Open Future," Disputatio 1 (19), 223-232 (2005)

40. Øhrstrøm P. "In Defence of the Thin Red Line: A Case for Ockhamism," Humana Mente 3, 8 (2009)

41. Pearle P. "Reduction of statevector by a nonlinear Schrödinger equation," Physical Review D, 13, 857 (1976)

42. Pooley O. "Relativity, the open future, and the passage of time," Proceedings of the Aristotelian Society, 113, 321-63 (2013)

43. Reichenbach H., The Direction of Time, Berkeley, University of Los Angeles Press (1956)

44. Sider T. Fourdimensionalism, Oxford, OUP (2001)

45. Tallant J. \& Ingram D. "A Defence of Lucretian Presentism", Australasian Journal of Philosophy (online first) (2020)

46. Thomasson R. H. "Indeterminist time and truth-value gaps," Theoria, 36, 264-281 (1970)

47. Torrengo G. "Time Travel and the Thin Red Line," in P. Graziani and M. Sangoi (eds.), Open Problems in the Philosophy of Science, London, College Publications, 245$258(2013)$

48. Wallace D., "What is Orthodox Quantum Mechanics?", https://arxiv.org/abs/1604.05973, (2016)

49. Wolff J. "Spin as a determinable?," Topoi, 34, 379-386 (2015)

50. Wilson J. M. "A Determinable-based Account of Metaphysical Indeterminacy," Inquiry, 56, 359-385 (2013) 